\title{
CONCERNING THE ANGLES AND THE ANGULAR DETERMINATION OF PLANES IN 4-SPACE.
}

\author{
BY DR. C. J. KEYSER.
}

( Read before the American Mathematical Society, April 26, 1902.)

\section{Introductory: Angles of Two Planes.}

IT has been established by Jordan* that, if two linear point spaces $S_{a}$ and $S_{\alpha}{ }^{\prime}$, each of $n-\alpha$ dimensions, in a linear point space of $n$ dimensions, have one and but one point in common, $S_{a}$ and $S_{\alpha}{ }^{\prime}$ form $\alpha$ angles. In the special case where $n=4$ and $\alpha=2, S$ and $S^{\prime}$ denote two planes of 4space, and, as two planes in 4-space have one and in general but one common point, it follows that two planes of 4-space form two angles. That such is the case can be readily seen independently of the general theorem mentioned, in either of the following reciprocal ways : Two planes $\pi$ and $\pi^{\prime}$ may be conceived as two flat pencils of lines having the common point $P$ of the planes as common vertex. Of all the angles formed by lines of the one pencil with lines of the other there is a least angle $\theta$, and this is not zero since by hypothesis the pencils have no line in common. Denote by $\pi^{\prime \prime}$ the plane determined by the lines $l$ and $l^{\prime}$ where the latter belong respectively to $\pi$ and $\pi^{\prime}$ and form the angle $\theta$. As the planes $\pi^{\prime}$ and $\pi^{\prime \prime}$ have the line $l^{\prime}$ in common, they form an ordinary angle $\alpha$. In order to bring $\pi$ and $\pi^{\prime}$ into coincidence, it is sufficient to turn $l^{\prime}$ in $\pi^{\prime \prime}$ through the angle $\theta$ and then, as $l$ and $l^{\prime}$ now coincide and $\pi$ and $\pi^{\prime}$ have a common line, to rotate $\pi$ or $\pi^{\prime}$ through the ordinary angle $\omega$ formed by $\pi$ with $\pi^{\prime}$ in its new position. Reciprocally, $\pi$ and $\pi^{\prime}$ may be regarded as pencils of lineoids $\dagger$ (ordinary 3-spaces). Either plane will accordingly be determined by any pair of its generating lineoids, i.e., the lineoids enveloping or containing it. The planes $\pi$ and $\pi^{\prime}$ have no common lineoid, for otherwise they would have, contrary to hypothesis, a common line. Accordingly there is a minimal angle $\varphi$, not zero, in the assemblage of angles formed by lineoids of the one plane with those of the other. Denote by $\pi^{\prime \prime}$ the plane determined by $L$ and $L^{\prime}$, where $L$ and $L^{\prime}$ are lineoids of $\pi$ and $\pi^{\prime}$ respectively and

* Jordan: "Essai sur la géométrie à n dimensions," Rull. de la Soc. Math. de France, vol. 3, p. 129.

† Cf. Cole : "On rotations in space of four dimensions," Amer. Jour. of Muth., 10'. 12. 
form the angle $\varphi$. To bring $\pi$ and $\pi^{\prime}$ into coincidence it is sufficient to rotate $L^{\prime}$ about $\pi^{\prime \prime}$ through the angle $\varphi$, which will cause $\pi$ and $\pi^{\prime}$ to lie in a same lineoid $L$ and so to intersect in a line, and then to turn $\pi^{\prime}$ or $\pi$ about this line through the angle $\gamma$ formed by $\pi$ with $\pi^{\prime}$ in its new position.

Hoppe * has shown that $\theta=\varphi$ and $\omega=\gamma$. The angles between two planes of 4-space may, therefore, receive the following equivalent reciprocal definitions: (1) The minimal angle $\theta$ between a lineoid $L$ of the one plane and a lineoid $L^{\prime}$ of the other, and the angle $\omega$ formed by the planes when they have been made collineoidal by the rotation of $L$ or $L^{\prime}$ about their common plane through the angle $\theta$; $(2)$ the minimal angle $\theta$ between a line $l$ of the one pencil (plane) and a line $l^{\prime}$ of the other, and the angle $\omega$ formed by the planes when they have been made collinear by rotating $l$ or $l^{\prime}$ in their common plane through the angle $\theta$.

A point (of 4-space) regarded as a plenum of planes is 4-dimensional. The plane geometry of this assemblage is of considerable interest both on its own account and on account of its analytical similarity to several other geometric theories, such as the Plücker line geometry, the sphere geometry of ordinary space, and particularly the geometry of the sphere surface which arises on employing as element the pencil of circles, $i$. e., an assemblage of circles of the sphere which have a pair of points in common. It is the object of this note to construct by means of the angles $\theta$ and $\omega$ a system of homogeneous coördinates, analogous to the Plücker coördinates, for the plane regarded as element of a 4-space point.

\section{Homogeneous Coördinates of the Plane.}

Without loss of generality we may choose the point in question for origin of rectangular point coördinates $x_{i}(i=$ $1,2,3,4)$. Assuming four mutually perpendicular lineoids, $x_{i}=0$, for coördinate lineoids, these will determine six planes and four lines.

Any plane $\pi$ of the assemblage of generating planes of the origin may be determined by a pair of lineoids

$$
\left\{\begin{array}{l}
\sum a_{i} x_{i}=0, \\
\sum b_{i} x_{i}=0,
\end{array} \quad(i=1,2,2,4) .\right.
$$

\footnotetext{
* Hoppe: "Ueber die Stellung der Ebene in der Vierdimensionengeometrie," Archiv d. Math. u. Physik., vol. 68 .
} 
The plane is equally determined by any two lineoids of the pencil

$$
\Sigma\left(\lambda a_{i}+\mu b_{i}\right) x_{i}=0
$$

of generating lineoids of $\pi$, and, in particular, by any pair of the special lineoids corresponding to the parametric values

$$
\mu: \lambda=-a_{i}: b_{i} \quad(i=1,2,3,4) .
$$

The equations of these lineoids are

$$
\left\{\begin{array}{r}
0 . x_{1}+p_{12} x_{2}+p_{13} x_{3}+p_{14} x_{4}=0 \\
-p_{12} x_{1}+0 . x_{2}+p_{23} x_{3}+p_{24} x_{4}=0 \\
-p_{13} x_{1}-p_{23} x_{2}+0 . x_{3}+p_{34} x_{4}=0 \\
-p_{13} x_{1}-p_{24} x_{2}-p_{34} x_{3}+0 . x_{4}=0
\end{array}\right.
$$

where $p_{i k} \equiv \alpha_{i} b_{k}-b_{i} a_{k}$.

These lineoids are geometrically distinguished among the generating lineoids of $\pi$ by the fact that they each contain a coördinate line. The quantities $p$ entering any pair of the equations (3) furnish four ratios, which are sufficient to determine the plane. The entire six $p$ 's furnish five ratios, which are, however, equivalent to four, by virtue of the identity

$$
p_{12} p_{34}+p_{13} p_{42}+p_{14} p_{23}=0 \text {. }
$$

The functions $p$ satisfy, moreover, the relations

$$
p_{i i}=0, \quad p_{i k}=-p_{k i} \text {. }
$$

The ratios are independent of the choice of defining lineoids, for if $\pi$ be supposed given by the lineoids, $\lambda_{1} a_{i}+$ $\mu_{1} b_{i}$ and $\lambda_{2} a_{i}+\mu_{2} b_{i}$, the corresponding quantities $p_{i k}{ }^{\prime}$ will be

$$
p_{i k}^{\prime}=\left(\lambda_{1} \mu_{2}-\lambda_{2} \mu_{1}\right) p_{i k^{\prime}}
$$

It can be readily shown that, conversely, any six functions $p_{i k}$ that satisfy the relations (4) and (5) will, if employed as in equations (3), determine four lineoids, enveloping, all of them, a same plane of the assemblage in question.

Accordingly, we may retain for coördinates of the plane, regarded as envelope of lineoids, six variables $p_{i k}$ which are subject to the relations (4) and (5). It will be sufficient to mention in passing that there is a reciprocal system of coör- 
dinates for the plane conceived as a pencil of lines. These coördinates will be proportional to the six coefficients $q_{i k}$ of the four equations representing the four lines which the plane has in common with the coördinate lineoids. These two coördinate systems correspond to and are formally identical with the Strahlercoordinaten and Axencoordinaten of Plücker.

III. Trigonometric Interpretation of the Coördinates $p_{i k}{ }^{\prime}$.

Suppose two planes $\pi$ and $\pi^{\prime}$ to be given by the two pairs of lineoids

$$
\left\{\begin{array}{l}
\sum \xi_{i} x_{i}=0, \quad \sum \xi_{i}^{\prime} x_{i}=0, \\
\sum \eta_{i} x_{i}=0, \quad \sum \eta^{\prime} x_{i}=0,
\end{array} \quad(i=1,2,3,4) .\right.
$$

If we assume, which may be done without loss of generality, that the equations (6) are in the normal form and also that the lineoids $\xi_{i}$ and $\eta_{i}$ as also $\xi_{i}^{\prime}$ and $\eta_{i}{ }^{\prime}$ are perpendicular, then

$$
\begin{gathered}
\sum \xi_{i}^{2}=\sum \eta_{i}{ }^{2}=\Sigma \xi_{i}^{\prime 2}=\sum \eta_{i}^{\prime 2}=1, \\
\sum \xi_{i} \eta_{i}=\Sigma \xi_{i}^{\prime} \eta_{i}^{\prime}=0 .
\end{gathered}
$$

In the paper already cited Hoppe shows that $\cos \theta$ and $\cos \omega$, where ${ }^{A}$ and $\omega$ are the angles of $\pi$ and $\pi^{\prime}$, are connected by the relation

(9) $\cos \theta \cos \omega=\sum \xi_{i} \xi_{i}^{\prime} \sum \eta_{i} \eta_{i}^{\prime}-\sum \xi_{i} \eta_{i}^{\prime} \sum \eta_{i} \xi_{i}^{\prime} \quad(i=1,2,3,4)$.

We have $\theta=\omega=90^{\circ}$, and then $\pi$ and $\pi^{\prime}$ are said to be absolutely perpendicular, when and only when

$$
\sum \xi_{i} \xi_{i}^{\prime}=\sum \eta_{i} \eta_{i}^{\prime}=\sum \xi_{i} \eta_{i}^{\prime}=\sum \eta_{i} \xi_{i}^{\prime}=0
$$

$i$. e., when and only when every generating lineoid of the one plane is perpendicular to every generating lineoid of the other. It follows that the planes of each of the pairs $\left(\pi_{12}\right.$, $\left.\pi_{34}\right),\left(\pi_{13}, \pi_{24}\right),\left(\pi_{14}, \pi_{23}\right)$ of coördinate planes, where $\pi_{\alpha \beta}$ stands for the plane determined by the coördinate lineoids $x_{a}=0$, $x_{\beta}=0$, are absolutely perpendicular. If, now, we denote by $\theta_{a \beta}$ and $\omega_{a \beta}$ the angles of the plane $\pi_{a \beta}$ with the plane $\pi$

$$
\left\{\begin{array}{l}
\sum a_{i} x_{i}=0 \\
\sum b_{i} x_{i}=0
\end{array}\right.
$$

where

$$
\sum a_{i} b_{i}=0, \quad \sum a_{i}^{2}=\Sigma b_{i}^{2}=1,
$$


we have the relations

$$
\begin{cases}\cos \theta_{12} \cos \omega_{12}=p_{12}, & \cos \theta_{34} \cos \omega_{34}=p_{34} \\ \cos \theta_{13} \cos \omega_{13}=p_{13}, & \cos \theta_{24} \cos \omega_{24}=p_{24} \\ \cos \theta_{14} \cos \omega_{14}=p_{14}, & \cos \theta_{23} \cos \omega_{23}=p_{23}\end{cases}
$$

where $p_{\alpha \beta}=a_{\alpha} b_{\beta}-b_{a} \alpha_{\beta}$, and the relation

$$
\begin{gathered}
\cos \theta_{12} \cos \omega_{12} \cos \theta_{34} \cos \omega_{34}-\cos \theta_{13} \cos \omega_{13} \cos \theta_{24} \cos \omega_{24} \\
+\cos \theta_{14} \cos \omega_{14} \cos \theta_{23} \cos \omega_{23} \equiv 0 .
\end{gathered}
$$

It remains to determine the changes which the expressions for the cosine products undergo in case the assumption that the defining lineoids (6) are perpendicular is abandoned. In this case we may suppose $\pi$ defined by the pair of perpendicular lineoids,

and $\pi^{\prime}$ by the pair,

$$
\left\{\begin{array}{l}
\Sigma\left(\xi_{i}+\eta_{i}\right) x_{i}=0 \\
\Sigma\left(\xi_{i}-\eta_{i}\right) x_{i}=0
\end{array}\right.
$$

$$
\left\{\begin{array}{l}
\Sigma\left(\xi_{i}^{\prime}+\eta_{i}^{\prime}\right) x_{i}=0, \\
\Sigma\left(\xi_{i}^{\prime}-\eta_{i}^{\prime}\right) x_{i}=0 .
\end{array}\right.
$$

If now these equations be written in the normal form, the expression for $\cos \theta \cos \omega$ becomes after reduction

$$
\frac{\sum \xi_{i} \xi_{i}^{\prime} \sum \eta_{i} \eta_{i}^{\prime}-\sum \xi_{i} \eta_{i}^{\prime} \sum \eta_{i} \xi_{i}^{\prime}}{\sqrt{\left\{1-\left(\sum \xi_{i} \eta_{i}\right)^{2}\right\}\left\{1-\left(\sum \xi_{i}^{\prime} \eta_{i}^{\prime}\right)^{2}\right\}}} .
$$

Denoting the angles between the lineoids $\xi_{i}$ and $\eta_{i}, \xi_{i}^{\prime}$ and $\eta_{i}^{\prime}, \cdots$, by the symbols $(\xi, \eta),\left(\xi^{\prime}, \eta^{\prime}\right), \cdots$, we may write

(17) $\cos \theta \cos \omega=\frac{\cos \left(\xi, \xi^{\prime}\right) \cos \left(\eta, \eta^{\prime}\right)-\cos \left(\xi, \eta^{\prime}\right) \cos \left(\eta, \xi^{\prime}\right)}{\sin (\xi, \eta) \sin \left(\xi^{\prime}, \eta^{\prime}\right)}$.

If the lineoids $a_{i}$ and $b_{i}$ of equations (11) are supposed to be non-perpendicular, equations (12) assume the form

$$
\cos \theta_{\alpha \beta} \cos \omega_{\alpha \beta}=p_{\alpha \beta}: \sin (a, b) .
$$

Inasmuch, however, as only the ratios of the $p$ 's are essential, $\sin (a, b)$ may be disregarded or absorbed in $p_{a \beta}$.

It appears, therefore, that in studying the geometry of planes through a point of 4-space, it is possible, instead 
of referring the plane to lines or lineoids as coördinate elements, to determine the plane by reference to planes, as follows: if from the plane assemblage in question six planes $\pi_{1}, \cdots, \pi_{6}$ be chosen such that they furnish three pairs $\pi_{1} \pi_{6}, \pi_{2} \pi_{5}, \pi_{3} \pi_{4}$ of absolutely perpendicular planes, then we may take for homogeneous plane coördinates of a plane $\pi$ the six cosine products $\cos \theta_{a} \cos \omega_{\alpha}$, where $\theta_{\alpha}$ and $\omega_{a}$ are the angles of $\pi$ with the coördinate plane $\pi_{a}$.

The six coördinate planes intersect an arbitrary lineoid of 4-space in the six edges of a tetraedron, while the arbitrary plane $\pi$ cuts from the lineoid an equally arbitrary line. The angles $\theta$ and $\omega$ correspond to the distances and angles of the line with the tetraedral lines. The corresponding interpretation of the Plücker coördinates would accordingly be one in terms of these distances and angles.

We will close this note with the necessary and sufficient condition that two planes $\left({ }_{a}, \omega_{a}\right)$ and $\left(\theta_{a}{ }^{\prime}, \omega_{a}{ }^{\prime}\right)$ shall have a common line. It is

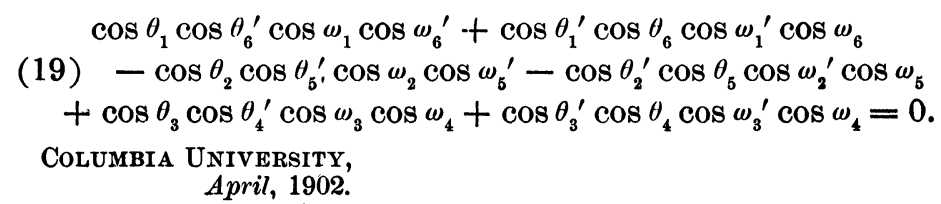

\section{NOTE ON THE SUFFICIENT CONDITIONS FOR AN ANALYTIC FUNCTION.}

BY MR. D. R. CURTISS.

(Read before the American Mathematical Society, April 26, 1902.)

Since the publication of Goursat's proof $*$ that a function of the complex variable $z$ possessing a derivative at each point of a two-dimensional region $T$ in which it is singlevalued must necessarily have a continuous derivative throughout that region, the question has arisen whether the sufficient conditions for an analytic function, stated in terms of the partial derivatives of the real and pure imaginary parts of the function, may not be reduced to simpler terms. These conditions are ordinarily given as follows:

$w=u(x, y)+i v(x, y)$ is an analytic function of the complex variable $z=x+i y$ at each point of a region $T$ of the $z$-plane if throughout $T$

\footnotetext{
* Trans. Am. Math. Soc., Vol. 1 (1900), No. 1, p. 14.
} 